\title{
Optimal Control of a Cancer Cell Model with Delay
}

\author{
C. Collins ${ }^{1}$, K.R. Fister ${ }^{2 *}$ and M. Williams ${ }^{3}$ \\ ${ }^{1}$ Department of Mathematics, University of Tennessee, Knoxville, TN 37996 USA \\ ${ }^{2}$ Department of Mathematics and Statistics, Murray State University, Murray, KY 42071 USA \\ ${ }^{3}$ Department of Mathematics, University of Nebraska, Lincoln, NE 68588 USA
}

\begin{abstract}
In this paper, we look at a model depicting the relationship of cancer cells in different development stages with immune cells and a cell cycle specific chemotherapy drug. The model includes a constant delay in the mitotic phase. By applying optimal control theory, we seek to minimize the cost associated with the chemotherapy drug and to minimize the number of tumor cells. Global existence of a solution has been shown for this model and existence of an optimal control has also been proven. Optimality conditions and characterization of the control are discussed.
\end{abstract}

Key words: cancer dynamics, optimal control

AMS subject classification: 34A12, 34H05

\section{Introduction}

Cells that can not regulate their own growth and division are classified as cancerous. Cancer cells that don't match the growth of normal tissue create an abnormal mass of tissue referred to as tumors. Liu et. al [13] takes a more extensive look at the development of cancer cells by focusing on three particular phases through which they travel. These stages are the mitotic-phase (dividing), quiescent phase (resting), and the interphase. The body's main defenses against tumors are the white blood cells, often called lymphocytes, generated by the immune system. Benign type tumor can be contained or it can grow into surrounding tissue. Tumors that grow into and destroy surrounding tissue are referred to as malignant. When either type of tumor begins to grow, it is common for them to be surgically removed and/or treated with chemotherapy.

\footnotetext{
*Corresponding author. E-mail: renee.fister@murraystate.edu
} 
Chemotherapy is the administering of anti-cancer drugs [2]. Numerous variables, such as toxicity, cause treatment to be highly complex. This complexity causes the strategy of treatment to vary widely from person to person. Immunotherapy is also another way to increase a patient's success at combating the cancer. This type of strategy is used to boost the immune system while other treatments are applied. Kirschner and Panetta [12] investigated the stability aspects of a combination therapy approach. It has proven quite useful to use mathematical modeling when determining strategies for fighting diseases which will enhance the quality of life for the patients. By using updated mathematical models, it is possible to make quantitative and testable predictions about real life patients. This enables a more accurate approach to treatments [14]. Fletcher [9] describes optimization as finding the 'best' solution to a mathematical problem. Fister and Donnelly [8] have analyzed an optimal control situation to find such a 'best' solution concept related to the Kirschner and Panetta work. Also, de Pillis et. al [4] has investigated optimal control strategies in chemoimmunotherapeutic treatment cases with traditional nonlinear controls in the objective functionals and with the opportunity to find combination controls of bang-bang and singular nature, [6].

Optimal control dates back to the 1950's and has often been applied to cancer therapy strategies. A study from 2000 uses optimal control techniques in designing drug protocols that kill a desired amount of tumor cells without killing the host, [1]. Works by Kim et. al [11], Swan and Vincent [17], and Murray [15] have successfully applied optimal control to maximize the effects of the chemotherapy drug while minimizing the toxicity and damage done by the drug. In this paper, we find the optimal strategy for minimizing the number of cancer cells and amount of chemotherapy drug needed in a model that includes a nonlinear relationship between immune cells and cancer cells. Ideally, we want to eliminate all the cancer cells and do as little damage to the body as possible.

The aforementioned study by Liu et. al [13] looks at the stability of a mitotic phase-specific drug and its interaction with the immune system and cancer cells, whose results show a significant decrease in the number of cancer cells but no change in the stability. A previous study by Villasana and Radunskaya [18] also considered the stability of using a cycle-specific drug and found that the stability depended on a delay of the cells moving from interphase into mitosis. It is important to note that the work in [18] did not look at the quiescent phase; whereas, Liu et. al [13] note that this phase greatly influences the cancer as a whole. The nondimensionalized model in [13] is the model this paper uses to conduct further study using optimal control techniques.

The arrangement of this paper starts with describing the model being used in Section 2. Section 3 gives context to the objective functional and establishes the existence of a solution to our problem given an optimal control in the admissible control set. Existence of an optimal control is derived there as well. Characterization of the controls is located in Section 4. A summary is provided in Section 5 .

\section{Model}

In this model, three phases of the cell cycle are considered; the quiescent phase where cells rest, the interphase where cells prepare for mitosis, and then the mitotic phase where cells divide. Liu 
et. al [13] developed the interaction between cells in the three phases, the immune system, and the cycle- specific drug. The variables used are as follows:

- $x$ - number of cancer cells in interphase phase

- $y$ - number of cancer cells in mitotic phase

- $z$-number of cancer cells in the quiescent phase

- $I$ - number of lymphocytes

- $u$ - biomass of chemotherapy drug in $\mathrm{mg}$

The model is:

$$
\begin{aligned}
\frac{d x}{d t}= & s \alpha_{3} z(t)-\alpha_{1} x(t)-\left(\sigma_{1}+k_{1} I(t)\right) x(t) \\
\frac{d y}{d t}= & \alpha_{1} x(t-\tau)-\left(\alpha_{2}+\sigma_{2}+k_{2} I(t)\right) y(t)-k_{4}\left(1-e^{-k_{5} u(t)}\right) y(t) \\
\frac{d z}{d t}= & 2 s^{-1} \alpha_{2} y(t)-\left(\alpha_{3}+\sigma_{3}+k_{3} I(t)\right) z(t) \\
\frac{d I}{d t}= & k+\left(\frac{\rho I(t)(x+y+s z)^{n}}{a+(x+y+s z)^{n}}\right)-\left(\sigma_{4}+c_{1} x(t)+c_{2} y(t)+c_{3} z(t)\right) I(t) \\
\frac{d u}{d t}= & -k_{6}\left(1-e^{-k_{7} u(t)}\right) I(t)
\end{aligned}
$$

where the initial conditions are

$$
x(t)=\phi(t), t \in[-\tau, 0], y(0)=y_{0}, z(0)=z_{0}, I(0)=I_{0}, u(0)=u_{0} .
$$

It is important to note that all the constants are positive and that the interphase is the only one with a delay present. Furthermore, this is a nondimensionalized system in which the parameter $s$ represents the number of initial cancer cells in the resting phase per number of initial cells in the interphase. The specific parameter values and a thorough description of the system can be found in Liu et. al [13]. Table 1 provides information about the meaning of the parameters. Information from Villasana and Radunskaya[18] gives insight into nonlinear growth, $\frac{\rho I(t)(x+y+s z)^{n}}{a+(x+y+s z)^{n}}$, in the concept that growth of immune cell populations are stimulated by the presence of cancer cells in a nonlinear manner. Values of $n$ have been estimated by $\frac{2}{3}$ and by positive integer values in $[4,5,18]$. Moreover, the destruction by drugs is shown by $\left(1-e^{-k_{5} u(t)}\right)$ and $\left(1-e^{-k_{7} u(t)}\right)$ in proportion to the mitotic cancer cell and lymphocyte populations, respectively [5]. The control term, $v(t)$, represents the inclusion of the chemotherapeutic drug as a source in the differential equation representing the quantification of such drug in the system. We also assume that $u_{0}>1$ as a condition to be used in the existence section. Also, $\phi(t)$ is a continuous, bounded function on $[-\tau, 0]$. 
Table 1: Meaning of parameters for system (2.1)

\begin{tabular}{|c|c|}
\hline Parameter & Parameter description \\
\hline$\alpha_{1}$ & rate at which cells move into mitosis \\
\hline$\alpha_{2}$ & rate at which cells move into the resting phase \\
\hline$\alpha_{3}$ & binding losses with immune cells \\
\hline$c_{i}$ for $i=1,2,3$ & natural death proportions for $x, y, z$, and $I$ \\
\hline$\sigma_{i}$ for $i=1,2,3,4$ & proportional growth of $I$ due to interaction with cancer cells \\
\hline$\rho$ & fractional exponent of growth from stimulus of cancer cells \\
\hline$n$ & rate at which $I$ reach saturation without stimulus \\
\hline$a$ & growth rate of $I$ with no cancer cells \\
\hline$k$ & rate $I$ destroys cells in different phases \\
\hline$k_{i}$ for $i=1,2,3$ & proportion of removal of $y$ and $I$ \\
\hline$k_{4}, k_{6}$ & proportion of drugs in removal of $y$ and $I$ \\
\hline$k_{5}, k_{7}$ & natural decay rate of chemotherapy \\
\hline$\gamma$ & time of cells in interphase (delay variable) \\
\hline$\tau$ &
\end{tabular}

\section{Existence}

In this section, the existence of a given solution to the system (2.1-2.2) given an optimal control is analyzed. Additionally, the existence of optimal control is proven. For clarification, the objective functionals are detailed below. Note that since the differential equation system is nonlinear in regard to its state variables, the objective functionals maintain this nonlinearity in terms of the square of the control terms. This inclusion depicts the scenario that small doses of the chemotherapeutic drug produces little changes in the system; whereas, larger doses can produce significant effects.

\subsection{Objective Functionals}

We consider two objective functionals, one without salvage terms and the other with salvage terms. We seek to minimize the first objective functional

$$
J_{1}(v)=\int_{0}^{t_{f}}\left[\frac{\epsilon}{2} v^{2}(t)+x(t)+y(t)+s z(t)\right] d t
$$

over the set $\mathbb{V}=\left\{t \in\left[0, t_{f}\right] \mid 0 \leq v(t) \leq 1\right\}$. Here $\epsilon$ is a weight factor, representing the cost to the system and $x, y, z$ are the cancer cells in specific phases of the cell cycle. We are minimizing both the tumor cells and the cost associated with the chemotherapy drug. We will also minimize the second objective functional

$$
J_{2}(v)=\int_{0}^{t_{f}} \frac{\epsilon}{2} v^{2}(t) d t+\left[x\left(t_{f}\right)+y\left(t_{f}\right)+s z\left(t_{f}\right)\right]
$$


over the same set $\mathbb{V}$ and with the same weight factor $\epsilon$. The cost associated with drug is being minimized, but the cancer cells $x, y$, and $z$ are minimized only at the final time.

\subsection{Existence}

We establish the existence of a solution to the delay differential system using results from R.D. Driver's text [7]. For notational purposes, we use

$f(\vec{x})=\left(\begin{array}{c}s \alpha_{3} z(t)-\alpha_{1} x(t)-\left(\sigma_{1}+k_{1} I(t)\right) x(t) \\ \alpha_{1} x(t-\tau)-\left(\alpha_{2}+\sigma_{2}+k_{2} I(t)\right) y(t)-k_{4}\left(1-e^{-k_{5} u(t)}\right) y(t) \\ 2 s^{-1} \alpha_{2} y(t)-\left(\alpha_{3}+\sigma_{3}+k_{3} I(t)\right) z(t) \\ k+\left(\frac{\rho I(t)(x+y+s z)^{n}}{a+(x+y+s z)^{n}}\right)-\left(\sigma_{4}+c_{1} x(t)+c_{2} y(t)+c_{3} z(t)\right) I(t)-k_{6}\left(1-e^{-k_{7} u(t)}\right) I(t) \\ -\gamma u(t)+v(t)\end{array}\right)$

where $\vec{x}=(x, y, z, I, u)^{T}$. We also note that if $\chi$ is a function defined at least on $[t-\tau, t] \rightarrow R^{n}$, then we can define a new function $\chi_{t}:[\tau, 0] \rightarrow R^{n}$ by

$$
\chi_{t}(\sigma)=\chi(t+\sigma)
$$

for $-\tau \leq \sigma \leq 0$. Consequently, we can reconsider system in (2.1-2.2) in the form

$$
\vec{x}^{\prime}(t)=F\left(t, x_{t}(\sigma), y(t), z(t), I(t), u(t)\right)
$$

where $F:[0, \beta) \times C[(-\tau, 0), R] \times R^{4} \rightarrow R^{n}$ for $\beta \in\left[0, t_{f}\right)$. This basically says that for each $[t, \psi, y, z, I, u] \in[0, \beta] \times C[(-\tau, 0), R] \times R^{4}, F(t, \psi, y, z, I, u)$ must be a well defined point in $R^{n}$. Thus, for our system (2.1)-(2.2) to be equivalent, we note that $x_{t}(t-\tau-t)=x(t+t-\tau-t)=$ $x(t-\tau)$ by using the representation in equation (3.3). Hence $x_{t}(-\tau)=x(t-\tau)$ and we have a relationship between $F$ and $f$ as

$$
\begin{array}{r}
F\left(t, x_{t}(-\tau), y(t), z(t), I(t), u(t)\right)=f\left(t, x_{t}(t-\tau-t), y(t), z(t), I(t), u(t)\right) \\
=f(t, x(t-\tau), y(t), z(t), I(t), u(t)) .
\end{array}
$$

Using the notation in the representation (3.5) in the differential equation system (3.4), we understand that $x(0)=\phi(0)$.

We can further consider a solution to our problem in terms of an integral equation. We see that

$$
x(t)= \begin{cases}\phi(t), & \text { if }-\tau \leq t \leq 0 \\ \phi(0)+\int_{0}^{t} F\left(s, x_{s}(-\tau), y(s), z(s), I(s), u(s)\right) d s & \text { if } 0 \leq t<t_{f}\end{cases}
$$

where $F$ is defined above in equation (3.5). To obtain a unique solution given a control in our set $\mathbb{V}$, we first justify that the right hand side of the differential equation system has continuous partial derivatives. This will allow us to discuss a Lipschitz condition for $F$. We will use a lemma from ([7], pg. 261) to obtain a local Lipschitz condition. 
Lemma 1. If $f(\vec{x})$ has continuous first partial derivatives with respect to all but its first argument, then $f(\vec{x})$ is locally Lipschitz.

Proof. We analyze the first partials of the right hand side of our system. For simplicity, we let $w=\frac{\rho a n(x+y+s z)^{n-1}}{\left(a+(x+y+s z)^{n}\right)^{2}}, q=\frac{\rho(x+y+s z)^{n}}{a+(x+y+s z)^{n}}$ and $j=\sigma_{4}+c_{1} x+c_{2} y+c_{3} z$.

The Jacobian of $f(\vec{x})$ with respect to $\vec{x}$ is

$$
\left(\begin{array}{ccccc}
-\alpha_{1}-\sigma_{1}-k_{1} I(t) & 0 & s \alpha_{3} & -k_{1} x & 0 \\
\alpha_{1} & -\left(\alpha_{2}+\sigma_{2}+k_{2} I\right)-k_{4}\left(1-e^{-k_{5} u}\right) & 0 & -k_{2} y & -k_{4} k_{5} e^{-k_{5} u} y \\
0 & 2 s^{-1} \alpha_{2} & -\left(\alpha_{3}+\sigma_{3}+k_{3} I\right) & -k_{3} z & 0 \\
\left(w-c_{1}\right) I & \left(w-c_{2}\right) I & \left(s w-c_{3}\right) I & q-j-k_{6}\left(1-e^{-k_{7} u}\right) & -k_{6} k_{7} e^{-k_{7} u} I \\
0 & 0 & 0 & 0 & -\gamma
\end{array}\right)
$$

All the partials in this matrix are continuous. Thus $f(\vec{x})$ is locally Lipschitz.

Moreover, since $f$ is locally Lipschitz on $[0, \beta] \times R^{5} \rightarrow R^{n}$, then the functional $F$ mapping $[0, \beta) \times C[(-\tau, 0), R] \times R^{4} \rightarrow R^{n}$ is locally Lipschitz. To obtain a unique solution, we invoke the following theorem in the aforementioned text on page 308 , in relation to our problem.

Theorem 2. Let $F\left(t, x_{t}, y, z, I, u\right):\left[0, t_{f}\right) \times C[(-\tau, 0), R] \times R^{4} \rightarrow R^{n}$ be continuous and be locally Lipschitz. If

$$
\|F(t, \psi)\| \leq M(t)+N(t)\|\psi\|
$$

on $\left[0, t_{f}\right) \times C[(-\tau, 0), R] \times R^{4}$ where $M(t)$ and $N(t)$ are continuous, positive functions on $\left[0, t_{f}\right)$ and $\psi=\left(x_{t}, y, z, I, u\right)^{T}$, then the unique noncontinuable solution exists on the entire interval $\left[-\tau, t_{f}\right)$.

Proof. $F\left(t, x_{t}, y, z, I, u\right)$ has already been shown to be locally Lipschitz. Also, with $g_{1}(t)=$ $t-\tau$ and the right hand side of our differential equation system (2.1) being continuous, then $F\left(t, x_{t}(-\tau), y(t), z(t), I(t), u(t)\right)$ is a composition of continuous functions and hence is continuous on $\left[0, t_{f}\right)$. Lastly, we show $\|F(t, \psi)\| \leq M(t)+N(t)\|\psi\|$ on the prerequisite domain.

We consider supersolutions of $x(t), y(t), z(t), I(t)$ as $X, Y, Z$, and $I_{\max }$ respectively. Using the upper bound on our control, we find from the differential equation (2.2) that

$$
u(t)=\left(u_{0}-1\right) e^{-\gamma t} \leq u_{0} .
$$

Consequently, a set of supersolutions become

$$
\left(\begin{array}{c}
X \\
Y \\
Z \\
I_{\max }
\end{array}\right)^{\prime}=\left(\begin{array}{cccc}
0 & 0 & s \alpha_{3} & 0 \\
\alpha_{1} & k_{4} e^{-k_{5} u_{0}} & 0 & 0 \\
0 & 2 s^{-1} \alpha_{2} & 0 & 0 \\
0 & 0 & \rho+k_{6} e^{-k_{7} u_{0}} & 0
\end{array}\right)\left(\begin{array}{c}
X \\
Y \\
Z \\
I_{\max }
\end{array}\right)+\left(\begin{array}{l}
0 \\
0 \\
0 \\
k
\end{array}\right)
$$


Note that the matrix does not include the growth due to stimulus term because

$$
\frac{(x+y+s z)^{n}}{\left(a+(x+y+s z)^{n}\right)}=\frac{w}{a+w} \leq 1 \text { if } w=(x+y+s z)^{n}
$$

We see that via our transformation in equation (3.5) that $\|F(t, \psi)\| \leq\|f(t, x(t-\tau), y, z, I, u)\|$. Therefore, we have that ||$F(t, \psi)\|\leq M+N\| \psi \|$ where

$$
M=\left(\begin{array}{l}
0 \\
0 \\
0 \\
k
\end{array}\right) \text { and } N=\left(\begin{array}{cccc}
0 & 0 & s \alpha_{3} & 0 \\
\alpha_{1} & k_{4} e^{-k_{5} u_{0}} & 0 & 0 \\
0 & 2 s^{-1} \alpha_{2} & 0 & 0 \\
0 & 0 & \rho+k_{6} e^{-k_{7} u_{0}} & 0
\end{array}\right) \text {. }
$$

Since $\mathrm{M}$ and $\mathrm{N}$ are both positive and continuous functions under the assumption that the constants are positive, then by application of this theorem, we have the uniqueness of a solution on $\left[-\tau, t_{f}\right)$ given our control in our admissible control set.

\subsection{Existence of an Optimal Control}

We develop the existence of an optimal control subject to the system defined in equations (2.1)(2.2). We restrict our attention to the second objective functional, $J_{2}(v)$, since it involves salvage terms or cancer cell populations that are to be minimized at the final time. The theorem we use to prove the existence of an optimal control is located in the Appendix as Theorem 5. A similar analysis follows for $J_{1}(v)$.

For notational purposes, we define a target set as a family $\mathcal{T}$ of nonempty compact sets $T_{t} \subset R^{n}$ for $t \in\left[t_{0}, \beta\right]$. For a general concept, an admissible control $w(t)$ defined on $\left[t_{0}, t_{1}\right]$ transfers a function $\phi_{1} \in B V\left(\left[\alpha, t_{0}\right], S\right)$, where $S \subset R^{n}$ and $\alpha$ represents the delay term, to a target set $\mathcal{T}$, if the trajectory $x(t)=x\left(t, t_{0}, \phi_{1}, w\right)$, corresponding to the function $\phi_{1}(t)$ and the control $w(t)$ maintains $x\left(t_{1}\right) \in T_{t_{1}}$. We recognize that $x\left(t, t_{0}, \phi, w\right)$ acts as the right hand side of our system (2.1) with the initial function of $\phi_{1}(t)=\phi(t), t_{0}=0$, and $w(t)=v(t)$.

Theorem 3. There exists an optimal control in our admissible control set, $\mathbb{V}$, for $J_{2}(v)$.

Proof. First we note that the assumptions $A_{1}$ and $A_{2}$ in the Das and Sharma Theorem listed as Theorem 5 in the Appendix are satisfied, essentially because our problem is defined and continuous for all variables. It has also been shown that $f\left(t, x_{t}, y, z, I, u, v(t)\right)$ is bounded above in the proof of existence of a solution to our problem. Therefore, assumption $A_{3}$ is met. Since these assumptions are met, then we must show each of the remaining parts of Theorem 5 listed numerically are satisfied to establish the existence of an optimal control in $\mathbb{V}$ that minimizes the functional $J_{2}(v)$. Since the initial condition is noted as bounded then $\phi \in B V([-\tau, 0], R)$. In addition, since our solution is bounded and exists then we obtain that our target set is nonempty. Below, we show that the functional is upper semicontinuous. Also, our control being bounded, provides us with the change in our control being bounded by a constant. Next, we need to analyze the functional. We notice that the integrand of the functional is a continuous real function defined on $\left[0, t_{f}\right) \times R^{5} \times R$. Lastly, we establish the necessary semicontinuity and recognize that the set $\mathbb{V}$ is nonempty. 
We note that there exists a minimizing sequence $v^{n} \in \mathbb{V}$ such that

$$
\lim _{n \rightarrow \infty} J_{2}\left(v^{n}\right)=\inf _{v \in \mathbb{V}} J(v)
$$

Since we know there exists a solution to our system (2.1-2.2), we define $x^{n}=x\left(v^{n}\right), y^{n}=y\left(v^{n}\right)$, $z^{n}=z\left(v^{n}\right)$. We see that $x^{n}\left(t_{f}\right) \rightarrow x^{*}\left(t_{f}\right), y^{n}\left(t_{f}\right) \rightarrow y^{*}\left(t_{f}\right)$, and $z^{n}\left(t_{f}\right) \rightarrow z^{*}\left(t_{f}\right)$ pointwise.

Moreover, we have that $v^{n} \rightarrow v^{*}$ in $L^{2}\left(0, t_{f}\right)$ since $v^{n}$ is in $\mathbb{V}$. We analyze

$$
\liminf _{n \rightarrow \infty} J_{2}\left(v^{n}\right)=\liminf _{n \rightarrow \infty}\left\{\int_{0}^{t_{f}} \frac{\epsilon}{2}\left(v^{n}\right)^{2} d t+\left(x^{n}\left(t_{f}\right)+y^{n}\left(t_{f}\right)+s z^{n}\left(t_{f}\right)\right)\right\} .
$$

By Fatou's Lemma,

$$
\begin{gathered}
\liminf _{n \rightarrow \infty} J_{2}\left(v^{n}\right) \geq \int_{0}^{t_{f}} \frac{\epsilon}{2} \liminf _{n \rightarrow \infty}\left[v^{n}\right]^{2} d t+\left(x^{*}+y^{*}+s z^{*}\right) \\
\geq \int_{0}^{t_{f}} \frac{\epsilon}{2}\left(v^{*}\right) d t+\left(x^{*}+y^{*}+s z^{*}\right) .
\end{gathered}
$$

So

$$
\liminf _{n \rightarrow \infty} J_{2}\left(v^{n}\right) \leq \lim _{n \rightarrow \infty} J_{2}\left(v^{n}\right) \leq J_{2}(v)
$$

Thus $J_{2}\left(v^{*}\right) \leq J_{2}(v)$ with $\mathbb{V}$ being nonempty.

All the enumerated properties are fulfilled from Das and Sharma's theorem, thus there exists an optimal control that minimizes $J_{2}(v)$.

\section{Characterization of the Control}

In this section the representation for an optimal control is developed. Involved in this process is the derivation of the adjoint system and hence the optimality system which encompasses the state system, the adjoint system and the optimal control representation that hinges on the interaction of the adjoints and the state solutions. We first define the two Hamiltonians, $H_{1}$, which is associated with the objective functional $J_{1}(v)$, and $H_{2}$, which is associated with the second objective functional $J_{2}(v)$.

$$
\begin{aligned}
H_{1}= & x(t)+y(t)+s z(t)+\frac{\epsilon}{2} v^{2}(t) \\
& +\lambda_{1}\left[\left(-\left(\alpha_{1}+\sigma_{1}\right) x(t)+s \alpha_{3} z(t)-k_{1} x(t) I(t)\right)\right] \\
& +\lambda_{2}\left[\left(\alpha_{1} x(t-\tau)-\left(\alpha_{2}+\sigma_{2}\right) y(t)-k_{2} y(t) I(t)-k_{4}\left(1-e^{-k_{5} u(t)}\right) y(t)\right)\right] \\
& +\lambda_{3}\left[2 s^{-1} \alpha_{2} y(t)-\left(\alpha_{3}+\sigma_{3}\right) z(t)-k_{3} z(t) I(t)\right] \\
& \left.+\lambda_{4}\left[k+\frac{\rho I(t)(x+y+s z)^{n}}{\left(a+(x+y+s z)^{n}\right)}-\left(\sigma_{4}+c_{1} x(t)+c_{2} y(t)+c_{3} z(t)\right) I(t)-k_{6}\left(1-e^{-k_{7} u(t)}\right) I(t)\right)\right] \\
& +\lambda_{5}[-\gamma u(t)+v(t)]
\end{aligned}
$$


and

$$
\begin{aligned}
H_{2}= & \frac{\epsilon}{2} v^{2}(t) \\
& +\lambda_{1}\left[\left(-\left(\alpha_{1}+\sigma_{1}\right) x(t)+s \alpha_{3} z(t)-k_{1} x(t) I(t)\right)\right] \\
& +\lambda_{2}\left[\left(\alpha_{1} x(t-\tau)-\left(\alpha_{2}+\sigma_{2}\right) y(t)-k_{2} y(t) I(t)-k_{4}\left(1-e^{-k_{5} u(t)}\right) y(t)\right)\right] \\
& +\lambda_{3}\left[2 s^{-1} \alpha_{2} y(t)-\left(\alpha_{3}+\sigma_{3}\right) z(t)-k_{3} z(t) I(t)\right] \\
& \left.+\lambda_{4}\left[k+\frac{\rho I(t)(x+y+s z)^{n}}{\left(a+(x+y+s z)^{n}\right)}-\left(\sigma_{4}+c_{1} x(t)+c_{2} y(t)+c_{3} z(t)\right) I(t)-k_{6}\left(1-e^{-k_{7} u(t)}\right) I(t)\right)\right] \\
& +\lambda_{5}[-\gamma u(t)+v(t)]
\end{aligned}
$$

Theorem 4. Given an optimal control, $v^{*}(t)$, and solutions of the corresponding state system, there exist adjoint variables $\lambda_{i}$ for $i=1,2, \ldots 5$ satisfying the following:

$$
\begin{array}{r}
-\frac{\partial H}{\partial x}-\left.\frac{\partial H}{\partial x(t-\tau)}\right|_{t+\tau}=\lambda_{1}^{\prime}=-1+\lambda_{1}\left(\alpha_{1}+\sigma_{1}\right)+\lambda_{1} k_{1} I-\lambda_{4} \frac{\rho \operatorname{Ina}(x+y+s z)^{(n-1)}}{\left(a+(x+y+s z)^{n}\right)^{2}} \\
+\lambda_{4} c_{1} I-\left.\lambda_{2} \alpha_{2}\right|_{t+\tau} \\
\qquad \begin{array}{c}
\text { (4.1) } 0 \leq t \leq t_{f}-\tau \\
-\frac{\partial H}{\partial x_{t}}=\lambda_{1}^{\prime}=-1+\lambda_{1}\left(\alpha_{1}+\sigma_{1}\right)+\lambda_{1} k_{1} I-\lambda_{4} \frac{\rho \operatorname{Ina}(x+y+s z)^{(n-1)}}{\left(a+(x+y+s z)^{n}\right)^{2}}+\lambda_{4} c_{1} I \quad \\
\text { for } t_{f}-\tau \leq t \leq t_{f}
\end{array}
\end{array}
$$

For the last four adjoints, the interval for $t$ is $\left[0, t_{f}\right]$.

$$
\begin{aligned}
-\frac{\partial H}{\partial y} & =\lambda_{2}^{\prime}=-1+\lambda_{2} k_{2} I+\lambda_{2} k_{4}\left(1-e^{-k_{5} u}\right)+\lambda_{2}\left(\alpha_{2}+\sigma_{2}\right)-\lambda_{3}\left(2 s^{-1} \alpha_{2}\right) \\
& -\lambda_{4}\left(\left(\frac{a \rho I(t) n(x+y+s z)^{(n-1)}}{\left(a+(x+y+s z)^{n}\right)^{2}}\right)-c_{2} I\right) \\
-\frac{\partial H}{\partial z} & =\lambda_{3}^{\prime}=-s-\lambda_{1} s \alpha_{3}+\lambda_{3}\left(\alpha_{3}+\sigma_{3}+I(t) k_{3}\right) \\
& -\lambda_{4}\left(\frac{a \rho I(t) n a s(x+y+s z)^{(n-1)}}{\left(a+(x+y+s z)^{n}\right)^{2}}\right)-\lambda_{4} c_{3} I(t) \\
-\frac{\partial H}{\partial I} & =\lambda_{4}^{\prime}=\lambda_{1} k_{1} x+\lambda_{2} k_{2} y+\lambda_{3} k_{3} z \\
& -\lambda_{4}\left(\left(\frac{\rho(x+y+s z)^{n}}{a+(x+y+s z)^{n}}+\sigma_{4}+c_{1} x+c_{2} y+c_{3} z+k_{6}\left(1-e^{-k_{7} u}\right)\right)\right) \\
-\frac{\partial H}{\partial u} & =\lambda_{5}^{\prime}=\lambda_{2} k_{4} k_{5} e^{-k_{5} u(t)} y+\lambda_{4} k_{6} k_{7} e^{-k_{7} u(t)} I+\lambda_{5} \gamma
\end{aligned}
$$


with $\lambda_{i}\left(t_{f}\right)=0$ for $i=1,2, . .5$.

We note that the adjoint equations associated with the objective functional $J_{2}(v)$ are the same as for equations (4.1)-(4.6). However, the transversality conditions are $\lambda_{i}\left(t_{f}\right)=1$ for $i=1,2,3$ and $\lambda_{j}\left(t_{f}\right)=0$ for $j=4,5$.

Furthermore, $v^{*}(t)$ associated with either $J_{1}(v)$ or $J_{2}(v)$ can be represented by

$$
v^{*}(t)=\min \left(\max \left(0, \frac{-\lambda_{5}(t)}{\epsilon}\right), 1\right) .
$$

Proof. Although the Hamiltonians are slightly different, both objective functionals will have the same control characterizations. Using Kamien and Schwartz [10] in conjunction with Pontryagin's Maximum Principle [16], we obtain the adjoint differential equations and the transversality conditions. We note that the existence of the adjoint solutions can be determined through similar arguments as was accomplished in Section 3. To find the representation for $v(t)$, we analyze the necessary condition for optimality $\frac{\partial H_{i}}{\partial v}=0$ for $i=1,2$ on the interior of the set, to obtain $\epsilon v(t)+\lambda_{5}(t)=0$.

We use standard optimal control strategies to find our characterization of our optimal control, $v^{*}(t)$, as

$$
v^{*}(t)=\min \left(\max \left(0, \frac{-\lambda_{5}(t)}{\epsilon}\right), 1\right)
$$

\section{Conclusion}

This paper shows the existence of a unique solution given a bounded control for a cancer-immune cell model incorporating quiescent, mitotic, and interphase components of the cell cycle. Existence of an optimal control is established using theory from Das and Sharma[3]. Optimal control techniques applied to delay differential equations in conjunction with Pontryagin's Maximum Principle [16] are used to determine the representation of the control through the calculation of the adjoint system that is coupled with the state system. Future work hinges on developing the numerical aspects of the optimal control simulations. Initial results have proven successful in matching the qualitative aspects given in Liu et. al [13]. Careful determination of the inclusion of the delay in the optimal control setting for the forward and backward oriented system in the numerical setting will be needed.

\section{Acknowledgements}

The work was supported by the National Science Foundation under Grant No. DMS-0531865, UBM, RUI: Biology and Mathematics in Population Studies (BioMaPS). Any opinions, findings, and conclusions or recommendations expressed in this material are those of the authors and do not necessarily reflect the views of the National Science Foundation,. We also thank Murray State 
University Undergraduate Research and Scholarly Activity Office for funds and undergraduate support.

\section{Appendix}

In this section, we give a statement of the theorem used for proving the existence of an optimal control.

Theorem 5 (Das and Sharma Theorem [3]). Let $\left[0, t_{f}\right]$ be a fixed interval and $S$ a domain on $R^{n}$. Let $B=\bigcup_{t \epsilon\left[0, t_{f}\right]} B V([\alpha, t], S)$. We shall denote by $V$ the set of all right continuous functions $v$ of bounded variation on $\left[0, t_{f}\right]$ into a nonempty compact subset of $Q$ of $R^{n}$.

Consider a control process governed by the measure delay-differential equation

$$
D x=f\left(t, x_{t}, y, z, I, u, v(t)\right)
$$

for $t \in\left[0, t_{f}\right]$ in which $f$ satisfies the following assumptions:

$A_{1}$ : The functional $f$ with range in $R^{n}$ is defined for all $t \epsilon\left[0, t_{f}\right]$, for all $x \in B$ and all $v \epsilon V$.

$A_{2}: f\left(t, x_{t}, y, z, I, u, v(t)\right)$ is continuous in $t, x_{t}, y, z, I, u$, and $v$.

$A_{3}$ : There exists a Lebesgue integrable real function $r(t)$ for $t \epsilon\left[0, t_{f}\right]$ such that

$$
\mid\left(f \left(t, x_{t}, y, z, I, u, v(t) \mid \leq r(t)\right.\right.
$$

uniformly with respect to $x \epsilon B$ and $v \epsilon V$.

Furthermore, the following conditions are met for the specified optimal control problem:

1. The initial function $\phi \in B V([\alpha, 0], S)$.

2. A target set $\mathcal{T}$ of nonempty compact sets $\mathcal{T}_{t} \subset R^{n}$ is defined on $\left[0, t_{f}\right]$ and is upper semicontinuous with respect to inclusion;

3. In the set $\mathbb{V}$ in $V$ of admissible controls $v(t)$ defined on subintervals contained in $\left[0, t_{f}\right]$ with the same left endpoint (and perhaps different right endpoints ) which transfer $\phi$ to $T$, it is such that for all $v \in V$

$|(\Delta v)| \leqq \Delta h$

on each subinterval of $\left[0, t_{f}\right]$, where $h$ is a given nondecreasing right continuous function defined on $\left[0, t_{f}\right]$, and

4. The integration of the objective functional is a continuous real function defined on $\left[0, t_{f}\right] \times$ $S \times Q$.

Then if the admissible set is nonempty, there exists an optimal control. 


\section{References}

[1] I. Athanassios, D. Barbolosi. Optimizing drug regimens in cancer chemotherapy by an efficacy-toxicity mathematical model. Comp. Biomedical Res., 33 (2000), 211-226.

[2] M. Chaplain, A. Matzavinos. Mathematical modelling of spatio-temporal phenomena in tumour immunology. Tutorials in Mathematical Biosciences III; Cell Cycle, Proliferation, and Cancer, 131-183, Springer-Verlag, Berlin, 2006.

[3] P. C. Das, R. R. Sharma. On optimal controls for measure delay-differential equations. SIAM J. Control, 6 (1971) No. 1, 43-61.

[4] L. G. de Pillis, K. R. Fister, W. Gu, T. Head, K. Maples, A. Murugan, T. Neal, K. Kozai. Optimal control of mixed immunotherapy and chemotherapy of tumors. Journal of Biological Systems, 16 (2008), No. 1, 51-80.

[5] L. G. de Pillis, K. R. Fister, W. Gu, C. Collins, M. Daub, D. Gross, J. Moore, B. Preskill. Mathematical Model Creation for Cancer Chemo-Immunotherapy. Computational and Mathematical Methods in Medicine, 10 (2009), No. 3, 165-184.

[6] L. G. de Pillis, K. R. Fister, W. Gu, C. Collins, M. Daub, D. Gross, J. Moore, B. Preskill. Seeking Bang-Bang Solutions of Mixed Immuno-chemotherapy of tumors. Electronic Journal of Differential Equations, (2007), No. 171, 1-24.

[7] R. D. Driver. Ordinary and Delay Differential Equations. Springer-Verlag, New York, 285311, 1977.

[8] K. R. Fister, J. H. Donnelly. Immunotherapy: An Optimal Control Theory Approach. Mathematical Biosciences in Engineering, 2 (2005), No. 3, 499-510.

[9] R. Fletcher. Practical methods of optimization. Wiley and Sons, New York, 1987.

[10] M. I. Kamien, N. L. Schwartz. Dynamic Optimization: The Calculus of Variations and Optimal Control in Economics and Management, Vol. 31 of Advanced Textbooks in Economics. North-Holland, 2nd edition, 1991.

[11] M. Kim, S. Perry, and K. B. Woo. Quantitative approach to the deisgn of antitumor drug dosage schedule via cell cycle kinetics and systems theory. Ann. Biomed. Eng., 5 (1977), $12-33$.

[12] D. Kirschner and J. C. Panetta. Modeling immunotherapy of the tumor-immune interaction. Journal of Mathematical Biology, 35 (1998), 235-252.

[13] W. Liu, T. Hillen, H. I. Freedman. A Mathematical model for M-phase specific chemotherapy including the $G_{o}$-phase and immunoresponse. Mathematical Biosciences and Engineering, 4(2007), No. 2, 239-259. 
[14] D. McKenzie. Mathematical modeling and cancer. SIAM News, 31, Jan/Feb 2004.

[15] J. M. Murray. Some optimality control problems in cancer chemotherapy with a toxicity limit. Mathematical Biosciences, 100 (1990), 49-67.

[16] L. S. Pontryagin, V. G. Boltyanksii, R. V. Gamkrelidze, E. F. Mischchenko. The Mathematical theory of optimal processes. Wiley, New York, 1962.

[17] G. W. Swan, T. L. Vincent. Optimal control analysis in the chemotherapy of IgG multiple myeloma. Bulletin of Mathematical Biology, 39 (1977), 317-337.

[18] M. Villasana, A. Radunskaya. A delay differential equation model for tumor growth. Journal of Mathematical Biology, 47(2003), 270-294. 